\title{
FUZZY TRANSLATIONS OF A FUZZY SET IN UP-ALGEBRAS
}

\author{
Tanintorn Guntasow ${ }^{1}$, Supanat Sajak ${ }^{2}$ Apirat Jomkham ${ }^{3}$, And \\ AIYARED IAMPAN ${ }^{4}$ \\ ${ }_{1,2,3,4}$ Department of Mathematics, School of Science, University of Phayao, \\ Phayao 56000, Thailand \\ ${ }^{4}$ aiyared.ia@up.ac.th
}

\begin{abstract}
In this paper, we apply the concept of fuzzy translations of a fuzzy set to UP-algebras. For any fuzzy set $\mu$ in a UP-algebra, the concepts of fuzzy $\alpha$ translations of $\mu$ of type I and of fuzzy $\beta$-translations of $\mu$ of type II are introduced, their basic properties are investigated and some useful examples are discussed. The concepts of prime fuzzy sets and of weakly prime fuzzy sets in UP-algebras are also studied. Moreover, we discuss the concepts of extensions and of intensions of a fuzzy set in UP-algebras.
\end{abstract}

Key words and Phrases: UP-algebra, fuzzy set, fuzzy translation, extension, intension.

\begin{abstract}
Abstrak. Di dalam paper ini, diaplikasikan konsep translasi fuzzy dari suatu himpunan fuzzy pada aljabar-UP. Untuk sebarang himpunan fuzzy $\mu$ di suatu aljabarUP, konsep $\alpha$-translasi fuzzy dari $\mu$ dengan tipe I dan konsep $\beta$-translasi dari $\mu$ dengan tipe II diperkenalkan, kemudian diinvestigasi beberapa sifat dasarnya, dan didiskusikan beberapa contoh penting. Konsep himpunan fuzzy prima dan himpunan fuzzy prima lemah di aljabar-UP juga dikaji di dalam paper ini. Lebih jauh, didiskusikan juga konsep ekstensi dan intensi dari suatu himpunan fuzzy di aljabarUP.
\end{abstract}

Kata kunci: aljabar-UP, himpunan fuzzy, translasi fuzzy, ekstensi, intensi.

2000 Mathematics Subject Classification: 03G25.

Received: 26 Nov 2016, revised: 15 March 2017, accepted: 16 March 2017. 


\section{Introduction and Preliminaries}

Among many algebraic structures, algebras of logic form important class of algebras. Examples of these are BCK-algebras [12], BCI-algebras [13], BCHalgebras [10], K-algebras [7], KU-algebras [21], SU-algebras [18] and others. They are strongly connected with logic. For example, BCI-algebras introduced by Iséki [13] in 1966 have connections with BCI-logic being the BCI-system in combinatory logic which has application in the language of functional programming. BCK and BCI-algebras are two classes of logical algebras. They were introduced by Imai and Iséki $[12,13]$ in 1966 and have been extensively investigated by many researchers. It is known that the class of BCK-algebras is a proper subclass of the class of BCI-algebras.

A fuzzy subset $f$ of a set $S$ is a function from $S$ to a closed interval $[0,1]$. The concept of a fuzzy subset of a set was first considered by Zadeh [25] in 1965. The fuzzy set theories developed by Zadeh and others have found many applications in the domain of mathematics and elsewhere. After the introduction of the concept of fuzzy sets by Zadeh [25], several researches were conducted on the generalizations of the notion of fuzzy set and application to many logical algebras such as: In 2001, Lele, Wu, Weke, Mamadou and Njock [20] studied fuzzy ideals and weak ideals in BCK-algebras. Jun [14] introduced the notion of Q-fuzzy subalgebras of BCK/BCIalgebras. In 2002, Jun, Roh and Kim [17] studied fuzzy B-algebras in B-algebras. In 2004, Jun [15] introduced the concept of $(\alpha, \beta)$-fuzzy ideals of BCK/BCI-algebras. In 2005, Akram and Dar [2] introduced the notions of $T$-fuzzy subalgebras and of $T$-fuzzy $H$-ideals in BCI-algebras. In 2007, Akram and Dar [3] introduced the notion of fuzzy ideals in K-algebras. In 2008, Akram [1] introduced the notion of bifuzzy ideals of K-algebras. In 2012, Sitharselvam, Priya and Ramachandran [23] introduced the notion of anti $Q$-fuzzy KU-ideals of KU-algebras. In 2016, Somjanta, Thuekaew, Kumpeangkeaw and Iampan [24] introduced and studied fuzzy UP-subalgebras and fuzzy UP-ideals of UP-algebras.

Moreover, fuzzy sets were extended to fuzzy translations in many logical algebras such as: In 2009, Lee, Jun and Doh [19] investigated relations among fuzzy translations, (normalized, maximal) fuzzy extensions and fuzzy multiplications of fuzzy subalgebras in BCK/BCI-algebras. In 2011, Jun [16] discussed fuzzy translations, fuzzy extensions and fuzzy multiplications of fuzzy ideals in BCK/BCIalgebras. In 2013, Chandramouleeswaran, Muralikrishna and Srinivasan [6] introduced the notions of fuzzy translations and fuzzy multiplications of fuzzy sets in BF-algebras. In 2014, Ansari and Chandramouleeswaran [4] introduced the notions of fuzzy translations, fuzzy extensions and fuzzy multiplications of fuzzy $\beta$-ideals of $\beta$-algebras. In 2015, Senapati, Bhowmik, Pal and Davvaz [22] discussed the fuzzy translations, fuzzy extensions and fuzzy multiplications of fuzzy H-ideals in BCK/BCI-algebras. Bhowmik, Senapati [5] introduced the notions of fuzzy translations, fuzzy extensions and fuzzy multiplications of fuzzy subalgebras in BGalgebras. In 2016, Hashemi [9] introduced the notions of fuzzy translations of fuzzy associative ideals in BCK/BCI-algebras. 
In this paper, we apply the concept of fuzzy translations of a fuzzy set to UPalgebras. For any fuzzy set $\mu$ in a UP-algebra, the concepts of fuzzy $\alpha$-translations of $\mu$ of type I and of fuzzy $\beta$-translations of $\mu$ of type II are introduced, their basic properties are investigated and some useful examples are discussed. The concepts of prime fuzzy sets and of weakly prime fuzzy sets in UP-algebras are also studied. Moreover, we discuss the concepts of extensions and of intensions of a fuzzy set in UP-algebras.

Before we begin our study, we will introduce the definition of a UP-algebra.

Definition 1.1. [11] An algebra $A=(A ; \cdot, 0)$ of type $(2,0)$ is called a UP-algebra if it satisfies the following axioms: for any $x, y, z \in A$,

$(\mathbf{U P}-\mathbf{1}):(y \cdot z) \cdot((x \cdot y) \cdot(x \cdot z))=0$,

(UP-2): $0 \cdot x=x$,

(UP-3): $x \cdot 0=0$, and

(UP-4): $x \cdot y=y \cdot x=0$ implies $x=y$.

From [11], we know that the notion of UP-algebras is a generalization of KU-algebras [21].

Example 1.2. [11] Let $X$ be a universal set. Define a binary operation $\cdot$ on the power set of $X$ by putting $A \cdot B=B \cap A^{\prime}=A^{\prime} \cap B=B-A$ for all $A, B \in \mathcal{P}(X)$. Then $(\mathcal{P}(X) ; \cdot, \emptyset)$ is a UP-algebra and we shall call it the power UP-algebra of type 1 .

Example 1.3. [11] Let $X$ be a universal set. Define a binary operation $*$ on the power set of $X$ by putting $A * B=B \cup A^{\prime}=A^{\prime} \cup B$ for all $A, B \in \mathcal{P}(X)$. Then $(\mathcal{P}(X) ; *, X)$ is a UP-algebra and we shall call it the power UP-algebra of type 2.

Example 1.4. Let $A=\{0,1,2,3\}$ be a set with a binary operation - defined by the following Cayley table:

\begin{tabular}{c|cccc}
$\cdot$ & 0 & 1 & 2 & 3 \\
\hline 0 & 0 & 1 & 2 & 3 \\
1 & 0 & 0 & 0 & 0 \\
2 & 0 & 1 & 0 & 3 \\
3 & 0 & 1 & 2 & 0
\end{tabular}

Then $(A ; \cdot, 0)$ is a UP-algebra.

In what follows, let $A$ and $B$ denote UP-algebras unless otherwise specified. The following proposition is very important for the study of UP-algebras.

Proposition 1.5. [11] In a UP-algebra A, the following properties hold: for any $x, y, z \in A$,

(1) $x \cdot x=0$

(2) $x \cdot y=0$ and $y \cdot z=0$ imply $x \cdot z=0$,

(3) $x \cdot y=0$ implies $(z \cdot x) \cdot(z \cdot y)=0$,

(4) $x \cdot y=0$ implies $(y \cdot z) \cdot(x \cdot z)=0$,

(5) $x \cdot(y \cdot x)=0$, 
(6) $(y \cdot x) \cdot x=0$ if and only if $x=y \cdot x$, and

(7) $x \cdot(y \cdot y)=0$.

On a UP-algebra $A=(A ; \cdot, 0)$, we define a binary relation $\leq$ on $A[11]$ as follows: for all $x, y \in A$,

$$
x \leq y \text { if and only if } x \cdot y=0 .
$$

Definition 1.6. [11] $A$ subset $S$ of $A$ is called a UP-subalgebra of $A$ if the constant 0 of $A$ is in $S$, and $(S ; \cdot, 0)$ itself forms a UP-algebra.

Proposition 1.7. [11] A nonempty subset $S$ of a UP-algebra $A=(A ; \cdot, 0)$ is a $U P$-subalgebra of $A$ if and only if $S$ is closed under the $\cdot$ multiplication on $A$.

Definition 1.8. [24] $A$ subset $F$ of $A$ is called a UP-filter of $A$ if it satisfies the following properties:

(1) the constant 0 of $A$ is in $F$, and

(2) for any $x, y \in A, x \cdot y \in F$ and $x \in F$ imply $y \in F$.

Definition 1.9. [11] $A$ subset $B$ of $A$ is called a UP-ideal of $A$ if it satisfies the following properties:

(1) the constant 0 of $A$ is in $B$, and

(2) for any $x, y, z \in A, x \cdot(y \cdot z) \in B$ and $y \in B$ imply $x \cdot z \in B$.

Definition 1.10. A subset $C$ of $A$ is called a strongly UP-ideal of $A$ if it satisfies the following properties:

(1) the constant 0 of $A$ is in $C$, and

(2) for any $x, y, z \in A,(z \cdot y) \cdot(z \cdot x) \in C$ and $y \in C$ imply $x \in C$.

Definition 1.11. [25] A fuzzy set in a nonempty set $X$ (or a fuzzy subset of $X$ ) is an arbitrary function $f: X \rightarrow[0,1]$ where $[0,1]$ is the unit segment of the real line.

Definition 1.12. [24] A fuzzy set $f$ in $A$ is called a fuzzy UP-subalgebra of $A$ if it satisfies the following property: for any $x, y \in A$,

$$
f(x \cdot y) \geq \min \{f(x), f(y)\} .
$$
all $x \in A$

By Proposition 1.51 , we have $f(0)=f(x \cdot x) \geq \min \{f(x), f(x)\}=f(x)$ for

Definition 1.13. [24] A fuzzy set $f$ in $A$ is called a fuzzy UP-filter of $A$ if it satisfies the following properties: for any $x, y \in A$,

(1) $f(0) \geq f(x)$, and

(2) $f(y) \geq \min \{f(x \cdot y), f(x)\}$

Definition 1.14. [24] A fuzzy set $f$ in $A$ is called a fuzzy UP-ideal of $A$ if it satisfies the following properties: for any $x, y, z \in A$,

(1) $f(0) \geq f(x)$, and

(2) $f(x \cdot z) \geq \min \{f(x \cdot(y \cdot z)), f(y)\}$. 
Definition 1.15. A fuzzy set $f$ in $A$ is called a fuzzy strongly UP-ideal of $A$ if it satisfies the following properties: for any $x, y, z \in A$,

(1) $f(0) \geq f(x)$, and

(2) $f(x) \geq \min \{f((z \cdot y) \cdot(z \cdot x)), f(y)\}$.

For example, we see that $\mu_{7}$ and $\mu_{8}$ are fuzzy strongly UP-ideal of $A$ in Example 3.23.

\section{Fuzzy $\alpha$-Translation of a Fuzzy Set}

In this section, we study the basic properties of UP-subalgebras (resp. UPfilters, UP-ideals, strongly UP-ideals) and fuzzy UP-subalgebras (resp. fuzzy UPfilters, fuzzy UP-ideals, fuzzy strongly UP-ideals) of a UP-algebra, and study the concept of prime and weakly prime of subsets and of fuzzy sets of a UP-algebra.

The proof of Theorems 2.1, 2.2, 2.4, and 2.6 can be verified easily.

Theorem 2.1. A subset $C$ of $A$ is a strongly UP-ideal of $A$ if and only if $C=A$.

Theorem 2.2. Every UP-filter of $A$ is a UP-subalgebra.

Example 2.3. Let $A=\{0,1,2,3\}$ be a set with a binary operation - defined by the following Cayley table:

\begin{tabular}{c|cccc}
$\cdot$ & 0 & 1 & 2 & 3 \\
\hline 0 & 0 & 1 & 2 & 3 \\
1 & 0 & 0 & 2 & 2 \\
2 & 0 & 1 & 0 & 2 \\
3 & 0 & 1 & 0 & 0
\end{tabular}

Hence, $(A ; \cdot, 0)$ is a UP-algebra. Let $S=\{0,2\}$. Then $S$ is a UP-subalgebra of $A$. Since $2 \cdot 3=2 \in S$, but $3 \notin S$, we have $S$ is not a UP-filter of $A$.

Theorem 2.4. Every UP-ideal of $A$ is a UP-filter.

Example 2.5. Let $A=\{0,1,2,3\}$ be a set with a binary operation - defined by the following Cayley table:

\begin{tabular}{c|cccc}
$\cdot$ & 0 & 1 & 2 & 3 \\
\hline 0 & 0 & 1 & 2 & 3 \\
1 & 0 & 0 & 2 & 2 \\
2 & 0 & 1 & 0 & 2 \\
3 & 0 & 1 & 0 & 0
\end{tabular}

Hence, $(A ; \cdot, 0)$ is a UP-algebra. Let $F=\{0,1\}$. Then $F$ is a UP-filter of $A$. Since $2 \cdot(1 \cdot 3)=0 \in F, 1 \in F$ but $2 \cdot 3=2 \notin F$, we have $F$ is not a UP-ideal of $A$.

Theorem 2.6. Every strongly UP-ideal of $A$ is a UP-ideal. 
Example 2.7. Let $A=\{0,1,2,3,4\}$ be a set with a binary operation $\cdot$ defined by the following Cayley table:

\begin{tabular}{c|ccccc}
$\cdot$ & 0 & 1 & 2 & 3 & 4 \\
\hline 0 & 0 & 1 & 2 & 3 & 4 \\
1 & 0 & 0 & 2 & 3 & 4 \\
2 & 0 & 0 & 0 & 3 & 4 \\
3 & 0 & 0 & 2 & 0 & 4 \\
4 & 0 & 0 & 0 & 0 & 0
\end{tabular}

Then $(A ; \cdot, 0)$ is a UP-algebra. Let $B=\{0,1,3\}$. Then $B$ is UP-ideal of $A$. Since $(2 \cdot 0) \cdot(2 \cdot 2)=0 \in B, 0 \in B$ but $2 \notin B$, we have $B$ is not a strongly UP-ideal of $A$.

By Theorems 2.2, 2.4, and 2.6 and Examples 2.3, 2.5, and 2.7, we have that the notion of UP-subalgebras is a generalization of UP-filters, the notion of UPfilters is a generalization of UP-ideals, and the notion of UP-ideals is a generalization of strongly UP-ideals.

Definition 2.8. [24] $A$ nonempty subset $B$ of $A$ is called a prime subset of $A$ if it satisfies the following property: for any $x, y \in A$,

$$
x \cdot y \in B \text { implies } x \in B \text { or } y \in B \text {. }
$$

Definition 2.9. [24] A UP-subalgebra (resp. UP-filter, UP-ideal, strongly UPideal) $B$ of $A$ is called a prime UP-subalgebra (resp. prime UP-filter, prime UPideal, prime strongly UP-ideal) of $A$ if it is a prime subset of $A$.

Definition 2.10. A nonempty subset $B$ of $A$ is called a weakly prime subset of $A$ if it satisfies the following property: for any $x, y \in A$ and $x \neq y$,

$$
x \cdot y \in B \text { implies } x \in B \text { or } y \in B \text {. }
$$

Example 2.11. Let $A=\{0,1,2,3\}$ be a set with a binary operation defined by the following Cayley table:

\begin{tabular}{c|cccc}
$\cdot$ & 0 & 1 & 2 & 3 \\
\hline 0 & 0 & 1 & 2 & 3 \\
1 & 0 & 0 & 2 & 3 \\
2 & 0 & 1 & 0 & 3 \\
3 & 0 & 1 & 2 & 0
\end{tabular}

Hence, $(A ; \cdot, 0)$ is a UP-algebra. Let $B=\{0,1\}$. Then $B$ is a weakly prime subset of $A$. Since $2 \cdot 2=0 \in B$, but $2 \notin B$, we have $B$ is not prime subset of $A$.

Definition 2.12. A UP-subalgebra (resp. UP-filter, UP-ideal, strongly UP-ideal) $B$ of $A$ is called a weakly prime UP-subalgebra (resp. weakly prime UP-filter, weakly prime UP-ideal, weakly prime strongly UP-ideal) of $A$ if it is a weakly prime subset of $A$.

The proof of Theorems 2.13, 2.14, 2.15, 2.17, and 2.19 and Lemma 2.21 can be verified easily.

Theorem 2.13. Let $S$ be a subset of $A$. Then the following statements are equivalent: 
(1) $S$ is a prime UP-subalgebra (resp. prime UP-filter, prime UP-ideal, prime strongly UP-ideal) of $A$,

(2) $S=A$, and

(3) $S$ is a strongly UP-ideal of $A$.

Theorem 2.14. A fuzzy set $f$ in $A$ is constant if and only if it is a fuzzy strongly $U P$-ideal of $A$.

Theorem 2.15. Every fuzzy UP-filter of $A$ is a fuzzy UP-subalgebra.

Example 2.16. Let $A=\{0,1,2,3\}$ be a set with a binary operation · defined by the following Cayley table:

\begin{tabular}{c|cccc}
$\cdot$ & 0 & 1 & 2 & 3 \\
\hline 0 & 0 & 1 & 2 & 3 \\
1 & 0 & 0 & 1 & 2 \\
2 & 0 & 0 & 0 & 1 \\
3 & 0 & 0 & 0 & 0
\end{tabular}

Then $(A ; \cdot, 0)$ is a UP-algebra. We define a mapping $f: A \rightarrow[0,1]$ as follows:

$$
f(0)=1, f(1)=0.6, f(2)=0.4 \text {, and } f(3)=0.1 \text {. }
$$

Then $f$ is a fuzzy UP-subalgebra of $A$. Since $f(2)=0.4<0.6=\min \{f(1 \cdot 2), f(1)\}$, we have $f$ is not a fuzzy UP-filter of $A$.

Theorem 2.17. Every fuzzy UP-ideal of $A$ is a fuzzy UP-filter.

Example 2.18. Let $A=\{0,1,2,3\}$ be a set with a binary operation - defined by the following Cayley table:

\begin{tabular}{c|cccc}
$\cdot$ & 0 & 1 & 2 & 3 \\
\hline 0 & 0 & 1 & 2 & 3 \\
1 & 0 & 0 & 2 & 2 \\
2 & 0 & 1 & 0 & 2 \\
3 & 0 & 1 & 0 & 0
\end{tabular}

Then $(A ; \cdot, 0)$ is a UP-algebra. We define a mapping $f: A \rightarrow[0,1]$ as follows:

$$
f(0)=1, f(1)=0.2, f(2)=0.1 \text {, and } f(3)=0.1 \text {. }
$$

Then $f$ is a fuzzy UP-filter of $A$. Since $f(2 \cdot 3)=0.1<0.2=\min \{f(2 \cdot(1 \cdot 3)), f(1)\}$, we have $f$ is not a fuzzy UP-ideal of $A$.

Theorem 2.19. Every fuzzy strongly UP-ideal of $A$ is a fuzzy UP-ideal.

Example 2.20. Let $A=\{0,1,2,3\}$ be a set with a binary operation $\cdot$ defined by the following Cayley table:

\begin{tabular}{c|cccc}
$\cdot$ & 0 & 1 & 2 & 3 \\
\hline 0 & 0 & 1 & 2 & 3 \\
1 & 0 & 0 & 2 & 3 \\
2 & 0 & 1 & 0 & 3 \\
3 & 0 & 1 & 2 & 0
\end{tabular}

Then $(A ; \cdot, 0)$ is a UP-algebra. We define a mapping $f: A \rightarrow[0,1]$ as follows: 


$$
f(0)=0.6, f(1)=0.4, f(2)=0.3 \text {, and } f(3)=0.2 .
$$

Then $f$ is a fuzzy UP-ideal of A. Since $f(1)=0.4<0.6=\min \{f((1 \cdot 0) \cdot(1$. $1)), f(0)\}$, we have $f$ is not a fuzzy strongly UP-ideal of $A$.

By Theorems 2.15, 2.17, and 2.19 and Examples 2.16, 2.18, and 2.20, we have that the notion of fuzzy UP-subalgebras is a generalization of fuzzy UP-filters, the notion of fuzzy UP-filters is a generalization of fuzzy UP-ideals, and the notion of fuzzy UP-ideals is a generalization of fuzzy strongly UP-ideals.

Lemma 2.21. If $f$ is a fuzzy UP-filter of $A$, then it is order preserving.

Definition 2.22. [24] A fuzzy set $f$ in $A$ is called a prime fuzzy set in $A$ if it satisfies the following property: for any $x, y \in A$,

$$
f(x \cdot y) \leq \max \{f(x), f(y)\} .
$$

Definition 2.23. [24] A fuzzy UP-subalgebra (resp. fuzzy UP-filter, fuzzy UP-ideal, fuzzy strongly UP-ideal) $f$ of $A$ is called a prime fuzzy UP-subalgebra (resp. prime fuzzy UP-filter, prime fuzzy UP-ideal, prime fuzzy strongly UP-ideal) of $A$ if it is a prime fuzzy set in A.

Definition 2.24. A fuzzy set $f$ in $A$ is called a weakly prime fuzzy set in $A$ if it satisfies the following property: for any $x, y \in A$ and $x \neq y$,

$$
f(x \cdot y) \leq \max \{f(x), f(y)\} .
$$

Example 2.25. Let $A=\{0,1,2,3\}$ be a set with a binary operation - defined by the following Cayley table:

\begin{tabular}{c|cccc}
$\cdot$ & 0 & 1 & 2 & 3 \\
\hline 0 & 0 & 1 & 2 & 3 \\
1 & 0 & 0 & 2 & 3 \\
2 & 0 & 1 & 0 & 3 \\
3 & 0 & 1 & 2 & 0
\end{tabular}

Then $(A ; \cdot, 0)$ is a UP-algebra. We define a mapping $f: A \rightarrow[0,1]$ as follows:

$$
f(0)=0.4, f(1)=0.3, f(2)=0.2 \text {, and } f(3)=0.1 \text {. }
$$

Then $f$ is a weakly prime fuzzy set of $A$. Since $f(1 \cdot 1)=f(0)=0.4>\max \{f(1)), f(1)\}=$ 0.3 , we have $f$ is not a prime fuzzy set of $A$.

Definition 2.26. A fuzzy UP-subalgebra (resp. fuzzy UP-filter, fuzzy UP-ideal, fuzzy strongly UP-ideal) $f$ of $A$ is called a weakly prime fuzzy UP-subalgebra (resp. weakly prime fuzzy UP-filter, weakly prime fuzzy UP-ideal, weakly prime fuzzy strongly UP-ideal) of $A$ if it is a weakly prime fuzzy set in $A$.

The proof of Theorems 2.27 , and 2.29 can be verified easily.

Theorem 2.27. Let $f$ be a fuzzy set in A. Then the following statements are equivalent:

(1) $f$ is a prime fuzzy UP-subalgebra (resp. prime fuzzy UP-filter, prime fuzzy $U P$-ideal, prime fuzzy strongly UP-ideal) of A, 
(2) $f$ is a constant fuzzy set in $A$, and

(3) $f$ is a fuzzy strongly UP-ideal of $A$.

Example 2.28. Let $A=\{0,1,2,3\}$ be a set with a binary operation - defined by the following Cayley table:

\begin{tabular}{c|cccc}
$\cdot$ & 0 & 1 & 2 & 3 \\
\hline 0 & 0 & 1 & 2 & 3 \\
1 & 0 & 0 & 2 & 3 \\
2 & 0 & 1 & 0 & 3 \\
3 & 0 & 1 & 2 & 0
\end{tabular}

Then $(A ; \cdot, 0)$ is a UP-algebra. We define a fuzzy set $\mu_{1}: A \rightarrow[0,1]$ in $A$ as follows:

$$
\mu_{1}(0)=0.6, \mu_{1}(1)=0.5, \mu_{1}(2)=0.5 \text {, and } \mu_{1}(3)=0.5 .
$$

Then $\mu_{1}$ is weakly prime fuzzy UP-subalgebras of A. Since $\mu_{1}(1 \cdot 1)=0.6>0.5=$ $\left.\max \left\{\mu_{1}(1)\right), \mu_{1}(1)\right\}$, we have $\mu_{1}$ is not a prime fuzzy UP-subalgebras of $A$.

We define a fuzzy set $\mu_{2}: A \rightarrow[0,1]$ in $A$ as follows:

$$
\mu_{2}(0)=0.6, \mu_{2}(1)=0.6, \mu_{2}(2)=0.5 \text {, and } \mu_{2}(3)=0.5 \text {. }
$$

Then $\mu_{2}$ is weakly prime fuzzy UP-filter of A. Since $\mu_{2}(2 \cdot 2)=0.6>0.5=$ $\left.\max \left\{\mu_{2}(2)\right), \mu_{2}(2)\right\}$, we have $\mu_{2}$ is not a prime fuzzy UP-filter of $A$.

We define a fuzzy set $\mu_{3}: A \rightarrow[0,1]$ in $A$ as follows:

$$
\mu_{3}(0)=0.6, \mu_{3}(1)=0.6, \mu_{3}(2)=0.6 \text {, and } \mu_{3}(3)=0.5 .
$$

Then $\mu_{3}$ is weakly prime fuzzy UP-ideal of $A$. Since $\mu_{3}(3 \cdot 3)=0.6>0.5=$ $\left.\max \left\{\mu_{3}(3)\right), \mu_{3}(3)\right\}$, we have $\mu_{3}$ is not a prime fuzzy UP-ideal of $A$.

Theorem 2.29. For UP-algebras, the notions of weakly prime fuzzy strongly UPideals and of prime fuzzy strongly UP-ideals coincide.

Definition 2.30. [8] The inclusion " $\subseteq$ " is defined by setting, for any fuzzy sets $\mu_{1}$ and $\mu_{2}$ in $A$,

$$
\mu_{1} \subseteq \mu_{2} \Leftrightarrow \mu_{1}(x) \leq \mu_{2}(x) \text { for all } x \in A .
$$

We say that $\mu_{2}$ is a fuzzy extension of $\mu_{1}$ [4], and $\mu_{1}$ is a fuzzy intension of $\mu_{2}$.

For any fuzzy set $\mu$ in $A$, we let $\dagger=1-\sup \{\mu(x) \mid x \in A\}$.

Definition 2.31. Let $\mu$ be a fuzzy set in $A$ and let $\alpha \in[0, \dagger]$. A mapping $\mu_{\alpha}^{\dagger}: A \rightarrow$ $[0,1]$ defined by

$$
\mu_{\alpha}^{\dagger}(x)=\mu(x)+\alpha \text { for all } x \in A
$$

is said to be a fuzzy $\alpha$-translation of $\mu$ of type I or, in short, a fuzzy $\alpha$-translation of $\mu$. 
Example 2.32. Let $A=\{0,1,2,3\}$ be a set with a binary operation $\cdot$ defined by the following Cayley table:

\begin{tabular}{c|cccc}
$\cdot$ & 0 & 1 & 2 & 3 \\
\hline 0 & 0 & 1 & 2 & 3 \\
1 & 0 & 0 & 1 & 2 \\
2 & 0 & 0 & 0 & 1 \\
3 & 0 & 0 & 0 & 0
\end{tabular}

Then $(A ; \cdot, 0)$ is a UP-algebra. We define a mapping $\mu: A \rightarrow[0,1]$ as follows:

$$
\mu(0)=0.7, \mu(1)=0.5, \mu(2)=0.3 \text {, and } \mu(3)=0.1 \text {. }
$$

Then $\dagger=1-\sup \{0.7,0.5,0.3,0.1\}=1-0.7=0.3$. Let $\alpha=0.2 \in[0,0.3]$. Then $a$ mapping $\mu_{\alpha}^{\dagger}: A \rightarrow[0,1]$ defined by

$$
\mu_{\alpha}^{\dagger}(0)=0.9, \mu_{\alpha}^{\dagger}(1)=0.7, \mu_{\alpha}^{\dagger}(2)=0.5, \text { and } \mu_{\alpha}^{\dagger}(3)=0.3
$$

The proof of Theorems 2.33, 2.34, 2.35, 2.36, 2.37, 2.38, 2.39, and 2.40 can be verified easily.

Theorem 2.33. If $\mu$ is a fuzzy UP-subalgebra of $A$, then the fuzzy $\alpha$-translation $\mu_{\alpha}^{\dagger}$ of $\mu$ is a fuzzy UP-subalgebra of $A$ for all $\alpha \in[0, \dagger]$.

Theorem 2.34. If there exists $\alpha \in[0, \dagger]$ such that the fuzzy $\alpha$-translation $\mu_{\alpha}^{\dagger}$ of $\mu$ is a fuzzy UP-subalgebra of $A$, then $\mu$ is a fuzzy UP-subalgebra of $A$.

Theorem 2.35. If $\mu$ is a fuzzy UP-filter of $A$, then the fuzzy $\alpha$-translation $\mu_{\alpha}^{\dagger}$ of $\mu$ is a fuzzy UP-filter of $A$ for all $\alpha \in[0, \dagger]$.

Theorem 2.36. If there exists $\alpha \in[0, \dagger]$ such that the fuzzy $\alpha$-translation $\mu_{\alpha}^{\dagger}$ of $\mu$ is a fuzzy UP-filter of $A$, then $\mu$ is a fuzzy UP-filter of $A$.

Theorem 2.37. If $\mu$ is a fuzzy UP-ideal of $A$, then the fuzzy $\alpha$-translation $\mu_{\alpha}^{\dagger}$ of $\mu$ is a fuzzy UP-ideal of $A$ for all $\alpha \in[0, \dagger]$.

Theorem 2.38. If there exists $\alpha \in[0, \dagger]$ such that the fuzzy $\alpha$-translation $\mu_{\alpha}^{\dagger}$ of $\mu$ is a fuzzy UP-ideal of $A$, then $\mu$ is a fuzzy UP-ideal of $A$.

Theorem 2.39. If $\mu$ is a fuzzy strongly UP-ideal of $A$, then the fuzzy $\alpha$-translation $\mu_{\alpha}^{\dagger}$ of $\mu$ is a fuzzy strongly UP-ideal of $A$ for all $\alpha \in[0, \dagger]$.

Theorem 2.40. If there exists $\alpha \in[0, \dagger]$ such that the fuzzy $\alpha$-translation $\mu_{\alpha}^{\dagger}$ of $\mu$ is a fuzzy strongly UP-ideal of $A$, then $\mu$ is a fuzzy strongly UP-ideal of $A$.

Theorem 2.41. If $\mu$ is a fuzzy UP-filter (resp. fuzzy UP-ideal, fuzzy strongly $U P$-ideal) of $A$, then the fuzzy $\alpha$-translation $\mu_{\alpha}^{\dagger}$ of $\mu$ is order preserving for all $\alpha \in[0, \dagger]$.

Proof. It follows from Theorem 2.35 (resp. Theorems 2.37 and 2.17, Theorems 2.39 and 2.19) and Lemma 2.21.

Theorem 2.42. If there exists $\alpha \in[0, \dagger]$ such that the fuzzy $\alpha$-translation $\mu_{\alpha}^{\dagger}$ of $\mu$ is a fuzzy UP-filter (resp. fuzzy UP-ideal, fuzzy strongly UP-ideal) of A, then $\mu$ is order preserving. 
Proof. It follows from Theorem 2.36 (resp. Theorems 2.38 and 2.17, Theorems 2.40 and 2.19) and Lemma 2.21.

Example 2.43. Let $A=\{0,1,2,3\}$ be a set with a binary operation - defined by the following Cayley table:

\begin{tabular}{c|cccc}
$\cdot$ & 0 & 1 & 2 & 3 \\
\hline 0 & 0 & 1 & 2 & 3 \\
1 & 0 & 0 & 2 & 3 \\
2 & 0 & 0 & 0 & 3 \\
3 & 0 & 0 & 0 & 0
\end{tabular}

Then $(A ; \cdot, 0)$ is a UP-algebra. We define a mapping $f: A \rightarrow[0,1]$ as follows:

$$
f(0)=1, f(1)=0.2, f(2)=0.3 \text {, and } f(3)=0.4 \text {. }
$$

Then $f$ is a fuzzy UP-subalgebra of $A$. Since $f(1)=0.2<\min \{f(2 \cdot 1)), f(2)\}=0.3$, we have $f$ is not a fuzzy UP-filter of $A$. Since $3 \leq 2$ but $f(3)=0.4>0.3=f(2)$, we have $f$ is not order preserving.

The proof of Theorems $2.44,2.45,2.46$, and 2.47 can be verified easily.

Theorem 2.44. If $\mu$ is a prime fuzzy set in $A$, then the fuzzy $\alpha$-translation $\mu_{\alpha}^{\dagger}$ of $\mu$ is a prime fuzzy set in $A$ for all $\alpha \in[0, \dagger]$.

Theorem 2.45. If there exists $\alpha \in[0, \dagger]$ such that the fuzzy $\alpha$-translation $\mu_{\alpha}^{\dagger}$ of $\mu$ is a prime fuzzy set in $A$, then $\mu$ is a prime fuzzy set in $A$.

Theorem 2.46. If $\mu$ is a weakly prime fuzzy set in $A$, then the fuzzy $\alpha$-translation $\mu_{\alpha}^{\dagger}$ of $\mu$ is a weakly prime fuzzy set in $A$ for all $\alpha \in[0, \dagger]$.

Theorem 2.47. If there exists $\alpha \in[0, \dagger]$ such that the fuzzy $\alpha$-translation $\mu_{\alpha}^{\dagger}$ of $\mu$ is a weakly prime fuzzy set in $A$, then $\mu$ is a weakly prime fuzzy set in $A$.

Theorem 2.48. If $\mu$ is a weakly prime fuzzy UP-subalgebra (resp. weakly prime fuzzy UP-filter, weakly prime fuzzy UP-ideal, weakly prime fuzzy strongly UP-ideal) of $A$, then the fuzzy $\alpha$-translation $\mu_{\alpha}^{\dagger}$ of $\mu$ is a weakly prime fuzzy UP-subalgebra (resp. weakly prime fuzzy UP-filter, weakly prime fuzzy UP-ideal, weakly prime fuzzy strongly UP-ideal) of $A$ for all $\alpha \in[0, \dagger]$.

Proof. It follows from Theorems 2.33 (resp. Theorem 2.35, Theorem 2.37, Theorem 2.39) and 2.46.

Theorem 2.49. If there exists $\alpha \in[0, \dagger]$ such that the fuzzy $\alpha$-translation $\mu_{\alpha}^{\dagger}$ of $\mu$ is a weakly prime fuzzy UP-subalgebra (resp. weakly prime fuzzy UP-filter, weakly prime fuzzy UP-ideal, weakly prime fuzzy strongly UP-ideal) of $A$, then $\mu$ is a weakly prime fuzzy UP-subalgebra (resp. weakly prime fuzzy UP-filter, weakly prime fuzzy $U P$-ideal, weakly prime fuzzy strongly UP-ideal) of $A$.

Proof. It follows from Theorems 2.34 (resp. Theorem 2.36, Theorem 2.38, Theorem 2.40 ) and 2.47 . 
Note 2.50. If $\mu$ is a fuzzy set in $A$ and $\alpha \in[0, \dagger]$, then $\mu_{\alpha}^{\dagger}(x)=\mu(x)+\alpha \geq \mu(x)$ for all $x \in A$. Hence, the fuzzy $\alpha$-translation $\mu_{\alpha}^{\dagger}$ of $\mu$ is a fuzzy extension of $\mu$ for all $\alpha \in[0, \dagger]$.

Lemma 2.51. Let $\mu$ and $\nu$ be fuzzy sets in $A$. If $\nu \supseteq \mu_{\beta}^{\dagger}$ for $\beta \in[0, \dagger]$, there exists $\alpha \in[0, \dagger]$ with $\alpha \geq \beta$ such that $\nu \supseteq \mu_{\alpha}^{\dagger} \supseteq \mu_{\beta}^{\dagger}$.

Proof. Assume that $\nu \supseteq \mu_{\beta}^{\dagger}$ for $\beta \in[0, \dagger]$. Then $\nu(x) \geq \mu_{\beta}^{\dagger}(x)$ for all $x \in A$. Putting $\alpha=\beta+\inf _{x \in A}\left\{\nu(x)-\mu_{\beta}^{\dagger}(x)\right\}$. Then

$$
\begin{aligned}
\inf _{x \in A}\left\{\nu(x)-\mu_{\beta}^{\dagger}(x)\right\} & =\inf _{x \in A}\{\nu(x)-(\mu(x)+\beta)\} \\
& \leq \inf _{x \in A}\{1-(\mu(x)+\beta)\} \\
& =1+\inf _{x \in A}\{-\mu(x)-\beta\} \\
& =1+\inf _{x \in A}\{-\mu(x)\}-\beta \\
& =1-\sup _{x \in A}\{\mu(x)\}-\beta \\
& =\dagger-\beta,
\end{aligned}
$$

so $\alpha=\beta+\inf _{x \in A}\left\{\nu(x)-\mu_{\beta}^{\dagger}(x)\right\} \leq \beta+\dagger-\beta=\dagger$. Thus $\alpha \in[0, \dagger]$ and $\alpha \geq \beta$, so $\mu_{\alpha}^{\dagger} \supseteq \mu_{\beta}^{\dagger}$. Now, for all $x \in A$, we have

$$
\begin{aligned}
\mu_{\alpha}^{\dagger}(x) & =\mu(x)+\alpha \\
& =\mu(x)+\beta+\inf _{x \in A}\left\{\nu(x)-\mu_{\beta}^{\dagger}(x)\right\} \\
& =\mu_{\beta}^{\dagger}(x)+\inf _{x \in A}\left\{\nu(x)-\mu_{\beta}^{\dagger}(x)\right\} \\
& \leq \mu_{\beta}^{\dagger}(x)+\nu(x)-\mu_{\beta}^{\dagger}(x) \\
& =\nu(x),
\end{aligned}
$$

so $\nu \supseteq \mu_{\alpha}^{\dagger}$. Hence, $\nu \supseteq \mu_{\alpha}^{\dagger} \supseteq \mu_{\beta}^{\dagger}$ for some $\alpha \in[0, \dagger]$ with $\alpha \geq \beta$.

Definition 2.52. Let $\mu_{1}$ and $\mu_{2}$ be two fuzzy sets in $A$ and $\mu_{1} \subseteq \mu_{2}$. If $\mu_{1}$ is a fuzzy UP-subalgebra (resp. fuzzy UP-filter, fuzzy UP-ideal, fuzzy strongly UP-ideal) of $A$, then $\mu_{2}$ is a fuzzy UP-subalgebra (resp. fuzzy UP-filter, fuzzy UP-ideal, fuzzy strongly UP-ideal) of $A$, and we say that $\mu_{2}$ is a fuzzy UP-subalgebra (resp. fuzzy UP-filter, fuzzy UP-ideal, fuzzy strongly UP-ideal) extension of $\mu_{1}$.

Theorem 2.53. If $\mu$ is a fuzzy UP-subalgebra (resp. fuzzy UP-filter, fuzzy UPideal, fuzzy strongly UP-ideal) of A, then the fuzzy $\alpha$-translation $\mu_{\alpha}^{\dagger}$ of $\mu$ is a fuzzy UP-subalgebra (resp. fuzzy UP-filter, fuzzy UP-ideal, fuzzy strongly UP-ideal) extension of $\mu$ for all $\alpha \in[0, \dagger]$.

Proof. It follows from Theorem 2.33 (resp. Theorem 2.35, Theorem 2.37, Theorem 2.39) and Note 2.50. 
Theorem 2.54. If $\mu$ is a fuzzy UP-subalgebra (resp. fuzzy UP-filter, fuzzy UPideal, fuzzy strongly UP-ideal) of $A$, then the fuzzy $\alpha$-translation $\mu_{\alpha}^{\dagger}$ of $\mu$ is a fuzzy UP-subalgebra (resp. fuzzy UP-filter, fuzzy UP-ideal, fuzzy strongly UP-ideal) extension of the fuzzy $\beta$-translation $\mu_{\beta}^{\dagger}$ of $\mu$ for all $\alpha, \beta \in[0, \dagger]$ with $\alpha \geq \beta$.

Proof. It follows from Theorem 2.33 (resp. Theorem 2.35, Theorem 2.37, Theorem 2.39).

Theorem 2.55. Let $\mu$ be a fuzzy UP-subalgebra (resp. fuzzy UP-filter, fuzzy UPideal, fuzzy strongly UP-ideal) of $A$ and $\beta \in[0, \dagger]$. For every fuzzy UP-subalgebra (resp. fuzzy UP-filter, fuzzy UP-ideal, fuzzy strongly UP-ideal) extension $\nu$ of the fuzzy $\beta$-translation $\mu_{\beta}^{\dagger}$ of $\mu$, there exists $\alpha \in[0, \dagger]$ with $\alpha \geq \beta$ such that $\nu$ is the fuzzy UP-subalgebra (resp. fuzzy UP-filter, fuzzy UP-ideal, fuzzy strongly UP-ideal) extension of the fuzzy $\alpha$-translation $\mu_{\alpha}^{\dagger}$ of $\mu$.

Proof. It follows from Theorem 2.33 (resp. Theorem 2.35, Theorem 2.37, Theorem 2.39) and Lemma 2.51.

\section{Fuzzy $\beta$-Translation of a Fuzzy Set}

For any fuzzy set $\mu$ in $A$, we let $\ddagger=\inf \{\mu(x) \mid x \in A\}$.

Definition 3.1. Let $\mu$ be a fuzzy set in $A$ and let $\beta \in[0, \ddagger]$. A mapping $\mu_{\beta}^{\ddagger}: A \rightarrow$ $[0,1]$ defined by

$$
\mu_{\beta}^{\ddagger}(x)=\mu(x)-\beta \text { for all } x \in A
$$

is said to be a fuzzy $\beta$-translation of $\mu$ of type II or, in short, a fuzzy $\beta$-translation of $\mu$.

Example 3.2. Let $A=\{0,1,2,3\}$ be a set with a binary operation - defined by the following Cayley table:

\begin{tabular}{l|llll}
$\cdot$ & 0 & 1 & 2 & 3 \\
\hline 0 & 0 & 1 & 2 & 3 \\
1 & 0 & 0 & 1 & 2 \\
2 & 0 & 0 & 0 & 1 \\
3 & 0 & 0 & 0 & 0
\end{tabular}

Then $(A ; \cdot, 0)$ is a UP-algebra. We define a mapping $\mu: A \rightarrow[0,1]$ as follows:

$$
\mu(0)=0.7, \mu(1)=0.5, \mu(2)=0.3 \text {, and } \mu(3)=0.1 \text {. }
$$

Then $\ddagger=\inf \{0.7,0.5,0.3,0.1\}=0.1$. Let $\beta=0.05 \in[0,0.1]$. Then a mapping $\mu_{\beta}^{\ddagger}: A \rightarrow[0,1]$ defined by

$$
\mu_{\beta}^{\ddagger}(0)=0.65, \mu_{\beta}^{\ddagger}(1)=0.45, \mu_{\beta}^{\ddagger}(2)=0.25 \text {, and } \mu_{\beta}^{\ddagger}(3)=0.05 .
$$

The proof of Theorems 3.3, 3.5, 3.6, 3.7, 3.8, 3.9, 3.10, and 3.11 can be verified easily.

Theorem 3.3. If $\mu$ is a fuzzy UP-subalgebra of $A$, then the fuzzy $\beta$-translation $\mu_{\beta}^{\ddagger}$ of $\mu$ is a fuzzy UP-subalgebra of $A$ for all $\beta \in[0, \ddagger]$. 
Example 3.4. In Example 2.16, a mapping $f: A \rightarrow[0,1]$ defined by

$$
f(0)=1, f(1)=0.6, f(2)=0.4 \text {, and } f(3)=0.1
$$

is a fuzzy UP-subalgebra of $A$. Then $\ddagger=\inf \{1,0.6,0.4,0.1\}=0.1$. For all $\beta \in$ $[0,0.1]$, we can show that a mapping $\mu_{\beta}^{\ddagger}: A \rightarrow[0,1]$ is a fuzzy UP-subalgebra of $A$.

Theorem 3.5. If there exists $\beta \in[0, \ddagger]$ such that the fuzzy $\beta$-translation $\mu_{\beta}^{\ddagger}$ of $\mu$ is a fuzzy UP-subalgebra of $A$, then $\mu$ is a fuzzy UP-subalgebra of $A$.

Theorem 3.6. If $\mu$ is a fuzzy UP-filter of $A$, then the fuzzy $\beta$-translation $\mu_{\beta}^{\ddagger}$ of $\mu$ is a fuzzy UP-filter of $A$ for all $\beta \in[0, \ddagger]$.

Theorem 3.7. If there exists $\beta \in[0, \ddagger]$ such that the fuzzy $\beta$-translation $\mu_{\beta}^{\ddagger}$ of $\mu$ is a fuzzy UP-filter of $A$, then $\mu$ is a fuzzy UP-filter of $A$.

Theorem 3.8. If $\mu$ is a fuzzy UP-ideal of $A$, then the fuzzy $\beta$-translation $\mu_{\beta}^{\ddagger}$ of $\mu$ is a fuzzy UP-ideal of $A$ for all $\beta \in[0, \ddagger]$.

Theorem 3.9. If there exists $\beta \in[0, \ddagger]$ such that the fuzzy $\beta$-translation $\mu_{\beta}^{\ddagger}$ of $\mu$ is a fuzzy UP-ideal of $A$, then $\mu$ is a fuzzy UP-ideal of $A$.

Theorem 3.10. If $\mu$ is a fuzzy strongly $U P$-ideal of $A$, then the fuzzy $\beta$-translation $\mu_{\beta}^{\ddagger}$ of $\mu$ is a fuzzy strongly UP-ideal of $A$ for all $\beta \in[0, \ddagger]$.

Theorem 3.11. If there exists $\beta \in[0, \ddagger]$ such that the fuzzy $\beta$-translation $\mu_{\beta}^{\ddagger}$ of $\mu$ is a fuzzy strongly UP-ideal of $A$, then $\mu$ is a fuzzy strongly UP-ideal of $A$.

Theorem 3.12. If $\mu$ is a fuzzy UP-filter (resp. fuzzy UP-ideal, fuzzy strongly $U P$-ideal) of $A$, then the fuzzy $\beta$-translation $\mu_{\beta}^{\ddagger}$ of $\mu$ is order preserving for all $\beta \in[0, \ddagger]$.

Proof. It follows from Theorem 3.6 (resp. Theorems 3.8 and 2.17, Theorems 3.10 and 2.19) and Lemma 2.21.

Theorem 3.13. If there exists $\beta \in[0, \dagger]$ such that the fuzzy $\beta$-translation $\mu_{\beta}^{\dagger}$ of $\mu$ is a fuzzy UP-filter (resp. fuzzy UP-ideal, fuzzy strongly UP-ideal) of $A$, then $\mu$ is order preserving.

Proof. It follows from Theorem 3.7 (resp. Theorems 3.9 and 2.17, Theorems 3.11 and 2.19) and Lemma 2.21.

The proof of Theorems 3.14, 3.15, 3.16, and 3.17 can be verified easily.

Theorem 3.14. If $\mu$ is a prime fuzzy set in $A$, then the fuzzy $\beta$-translation $\mu_{\beta}^{\ddagger}$ of $\mu$ is a prime fuzzy set in $A$ for all $\beta \in[0, \ddagger]$.

Theorem 3.15. If there exists $\beta \in[0, \ddagger]$ such that the fuzzy $\beta$-translation $\mu_{\beta}^{\ddagger}$ of $\mu$ is a prime fuzzy set in $A$, then $\mu$ is a prime fuzzy set in $A$.

Theorem 3.16. If $\mu$ is a weakly prime fuzzy set in $A$, then the fuzzy $\beta$-translation $\mu_{\beta}^{\ddagger}$ of $\mu$ is a weakly prime fuzzy set in $A$ for all $\beta \in[0, \ddagger]$. 
Theorem 3.17. If there exists $\beta \in[0, \ddagger]$ such that the fuzzy $\beta$-translation $\mu_{\beta}^{\ddagger}$ of $\mu$ is a weakly prime fuzzy set in $A$, then $\mu$ is a weakly prime fuzzy set in $A$.

Theorem 3.18. If $\mu$ is a weakly prime fuzzy UP-subalgebra (resp. weakly prime fuzzy UP-filter, weakly prime fuzzy UP-ideal, weakly prime fuzzy strongly UP-ideal) of $A$, then the fuzzy $\beta$-translation $\mu_{\beta}^{\ddagger}$ of $\mu$ is a weakly prime fuzzy UP-subalgebra (resp. weakly prime fuzzy UP-filter, weakly prime fuzzy UP-ideal, weakly prime fuzzy strongly UP-ideal) of $A$ for all $\beta \in[0, \ddagger]$.

Proof. It follows from Theorems 3.3 (resp. Theorem 3.6, Theorem 3.8, Theorem $3.10)$ and 3.16.

Theorem 3.19. If there exists $\beta \in[0, \ddagger]$ such that the fuzzy $\beta$-translation $\mu_{\beta}^{\ddagger}$ of $\mu$ is a weakly prime fuzzy UP-subalgebra (resp. weakly prime fuzzy UP-filter, weakly prime fuzzy UP-ideal, weakly prime fuzzy strongly UP-ideal) of $A$, then $\mu$ is a weakly prime fuzzy UP-subalgebra (resp. weakly prime fuzzy UP-filter, weakly prime fuzzy UP-ideal, weakly prime fuzzy strongly UP-ideal) of A.

Proof. It follows from Theorems 3.5 (resp. Theorem 3.7, Theorem 3.9, Theorem 3.11) and 3.17.

Note 3.20. If $\mu$ is a fuzzy set in $A$ and $\beta \in[0, \ddagger]$, then $\mu_{\beta}^{\ddagger}(x)=\mu(x)-\beta \leq \mu(x)$ for all $x \in A$. Hence, the fuzzy $\beta$-translation $\mu_{\beta}^{\ddagger}$ of $\mu$ is a fuzzy intension of $\mu$ for all $\beta \in[0, \ddagger]$.

Lemma 3.21. Let $\mu$ and $\nu$ be fuzzy sets in $A$. If $\nu \subseteq \mu_{\beta}^{\ddagger}$ for $\beta \in[0, \ddagger]$, there exists $\alpha \in[0, \ddagger]$ with $\alpha \geq \beta$ such that $\nu \subseteq \mu_{\alpha}^{\ddagger} \subseteq \mu_{\beta}^{\ddagger}$.

Proof. Assume that $\nu \subseteq \mu_{\beta}^{\ddagger}$ for $\beta \in[0, \ddagger]$. Then $\nu(x) \leq \mu_{\beta}^{\ddagger}(x)$ for all $x \in A$. Putting $\alpha=\beta+\inf _{x \in A}\left\{\mu_{\beta}^{\ddagger}(x)-\nu(x)\right\}$. Then

$$
\begin{aligned}
\inf _{x \in A}\left\{\mu_{\beta}^{\ddagger}(x)-\nu(x)\right\} & \leq \inf _{x \in A}\left\{\mu_{\beta}^{\ddagger}(x)\right\} \\
& =\inf _{x \in A}\{(\mu(x)-\beta)\} \\
& =\inf _{x \in A}\{\mu(x)\}-\beta \\
& =\ddagger-\beta,
\end{aligned}
$$


so $\alpha=\beta+\inf _{x \in A}\left\{\mu_{\beta}^{\ddagger}(x)-\nu(x)\right\} \leq \beta+\ddagger-\beta=\ddagger$. Thus $\alpha \in[0, \ddagger]$ and $\alpha \geq \beta$, so $\mu_{\alpha}^{\dagger} \subseteq \mu_{\beta}^{\dagger}$. Now, for all $x \in A$, we have

$$
\begin{aligned}
\mu_{\alpha}^{\ddagger}(x) & =\mu(x)-\alpha \\
& =\mu(x)-\left(\beta+\inf _{x \in A}\left\{\mu_{\beta}^{\ddagger}(x)-\nu(x)\right\}\right) \\
& =\mu(x)-\beta-\inf _{x \in A}\left\{\mu_{\beta}^{\ddagger}(x)-\nu(x)\right\} \\
& =\mu_{\beta}^{\ddagger}(x)+\sup _{x \in A}\left\{\nu(x)-\mu_{\beta}^{\ddagger}(x)\right\} \\
& \geq \mu_{\beta}^{\ddagger}(x)+\nu(x)-\mu_{\beta}^{\ddagger}(x) \\
& =\nu(x),
\end{aligned}
$$

so $\nu \subseteq \mu_{\alpha}^{\ddagger}$. Hence, $\nu \subseteq \mu_{\alpha}^{\ddagger} \subseteq \mu_{\beta}^{\ddagger}$ for some $\alpha \in[0, \ddagger]$ with $\alpha \geq \beta$.

Definition 3.22. Let $\mu_{1}$ and $\mu_{2}$ be two fuzzy sets in $A$ and $\mu_{1} \subseteq \mu_{2}$. If $\mu_{2}$ is a fuzzy UP-subalgebra (resp. fuzzy UP-filter, fuzzy UP-ideal, fuzzy strongly UP-ideal) of $A$, then $\mu_{1}$ is a fuzzy UP-subalgebra (resp. fuzzy UP-filter, fuzzy UP-ideal, fuzzy strongly UP-ideal) of $A$, and we say that $\mu_{1}$ is a fuzzy UP-subalgebra (resp. fuzzy UP-filter, fuzzy UP-ideal, fuzzy strongly UP-ideal) intension of $\mu_{2}$.

Example 3.23. Let $A=\{0,1,2,3\}$ be a set with a binary operation - defined by the following Cayley table:

\begin{tabular}{c|cccc}
$\cdot$ & 0 & 1 & 2 & 3 \\
\hline 0 & 0 & 1 & 2 & 3 \\
1 & 0 & 0 & 2 & 3 \\
2 & 0 & 1 & 0 & 3 \\
3 & 0 & 1 & 2 & 0
\end{tabular}

Then $(A ; \cdot, 0)$ is a UP-algebra. We define two fuzzy sets $\mu_{1}: A \rightarrow[0,1]$ and $\mu_{2}: A \rightarrow$ $[0,1]$ in $A$ as follows:

$$
\begin{aligned}
& \mu_{1}(0)=0.6, \mu_{1}(1)=0.4, \mu_{1}(2)=0.3, \text { and } \mu_{1}(3)=0.2, \\
& \mu_{2}(0)=0.9, \mu_{2}(1)=0.7, \mu_{2}(2)=0.6, \text { and } \mu_{2}(3)=0.5 .
\end{aligned}
$$

Then $\mu_{1} \subseteq \mu_{2}$, and $\mu_{1}$ and $\mu_{2}$ are fuzzy UP-subalgebras of $A$. Hence, $\mu_{1}$ is a fuzzy UP-subalgebra intension of $\mu_{2}$, and $\mu_{2}$ is a fuzzy UP-subalgebra extension of $\mu_{1}$. We define two fuzzy sets $\mu_{3}: A \rightarrow[0,1]$ and $\mu_{4}: A \rightarrow[0,1]$ in $A$ as follows:

$$
\begin{aligned}
& \mu_{3}(0)=0.5, \mu_{3}(1)=0.3, \mu_{3}(2)=0.1, \text { and } \mu_{3}(3)=0.5, \\
& \mu_{4}(0)=0.8, \mu_{4}(1)=0.6, \mu_{4}(2)=0.4, \text { and } \mu_{4}(3)=0.8 .
\end{aligned}
$$

Then $\mu_{3} \subseteq \mu_{4}$, and $\mu_{3}$ and $\mu_{4}$ are fuzzy UP-filter of A. Hence, $\mu_{3}$ is a fuzzy UPfilter intension of $\mu_{4}$, and $\mu_{4}$ is a fuzzy UP-filter extension of $\mu_{3}$. We define two fuzzy sets $\mu_{5}: A \rightarrow[0,1]$ and $\mu_{6}: A \rightarrow[0,1]$ in $A$ as follows:

$$
\begin{aligned}
& \mu_{5}(0)=0.6, \mu_{5}(1)=0.4, \mu_{5}(2)=0.3, \text { and } \mu_{5}(3)=0.2, \\
& \mu_{6}(0)=0.9, \mu_{6}(1)=0.7, \mu_{6}(2)=0.6, \text { and } \mu_{6}(3)=0.3 .
\end{aligned}
$$


Then $\mu_{5} \subseteq \mu_{6}$, and $\mu_{5}$ and $\mu_{6}$ are fuzzy UP-ideal of $A$. Hence, $\mu_{5}$ is a fuzzy UPideal intension of $\mu_{6}$, and $\mu_{6}$ is a fuzzy UP-ideal extension of $\mu_{5}$. We define two fuzzy sets $\mu_{7}: A \rightarrow[0,1]$ and $\mu_{8}: A \rightarrow[0,1]$ in $A$ as follows:

$$
\mu_{7}(x)=0.5 \text { and } \mu_{8}(x)=0.8 \text {. for all } x \in A \text {. }
$$

Then $\mu_{7} \subseteq \mu_{8}$, and $\mu_{7}$ and $\mu_{8}$ are fuzzy strongly UP-ideal of $A$. Hence, $\mu_{7}$ is a fuzzy strongly UP-ideal intension of $\mu_{8}$, and $\mu_{8}$ is a fuzzy strongly UP-ideal extension of $\mu_{7}$.

Theorem 3.24. If $\mu$ is a fuzzy UP-subalgebra (resp. fuzzy UP-filter, fuzzy UPideal, fuzzy strongly UP-ideal) of $A$, than the fuzzy $\beta$-translation $\mu_{\beta}^{\ddagger}$ of $\mu$ is a fuzzy UP-subalgebra (resp. fuzzy UP-filter, fuzzy UP-ideal, fuzzy strongly UP-ideal) intension of $\mu$ for all $\beta \in[0, \ddagger]$.

Proof. It follows from Theorem 3.3 (resp. Theorem 3.6, Theorem 3.8, Theorem 3.10) and Note 3.20.

Theorem 3.25. If $\mu$ is a fuzzy UP-subalgebra (resp. fuzzy UP-filter, fuzzy UPideal, fuzzy strongly UP-ideal) of $A$, then the fuzzy $\alpha$-translation $\mu_{\alpha}^{\ddagger}$ of $\mu$ is a fuzzy UP-subalgebra (resp. fuzzy UP-filter, fuzzy UP-ideal, fuzzy strongly UP-ideal) intension of the fuzzy $\beta$-translation $\mu_{\beta}^{\ddagger}$ of $\mu$ for all $\alpha, \beta \in[0, \ddagger]$ with $\alpha \geq \beta$.

Proof. It follows from Theorem 3.3 (resp. Theorem 3.6, Theorem 3.8, Theorem $3.10)$.

Theorem 3.26. Let $\mu$ be a fuzzy UP-subalgebra (resp. fuzzy UP-filter, fuzzy UPideal, fuzzy strongly UP-ideal) of $A$ and $\beta \in[0, \ddagger]$. For every fuzzy UP-subalgebra (resp. fuzzy UP-filter, fuzzy UP-ideal, fuzzy strongly UP-ideal) intension $\nu$ of the fuzzy $\beta$-translation $\mu_{\beta}^{\ddagger}$ of $\mu$, there exists $\alpha \in[0, \ddagger]$ with $\alpha \geq \beta$ such that $\nu$ is the fuzzy UP-subalgebra (resp. fuzzy UP-filter, fuzzy UP-ideal, fuzzy strongly UP-ideal) intension of the fuzzy $\alpha$-translation $\mu_{\alpha}^{\ddagger}$ of $\mu$.

Proof. It follows from Theorem 3.3 (resp. Theorem 3.6, Theorem 3.8, Theorem 3.10 ) and Lemma 3.21.

Theorem 3.27. If there exists $\alpha \in[0, \dagger]$ such that the fuzzy $\alpha$-translation $\mu_{\alpha}^{\dagger}$ of $\mu$ is a fuzzy UP-subalgebra (resp. fuzzy UP-filter, fuzzy UP-ideal, fuzzy strongly $U P$-ideal) of $A$, then the fuzzy $\beta$-translation $\mu_{\beta}^{\ddagger}$ of $\mu$ is a fuzzy UP-subalgebra (resp. fuzzy UP-filter, fuzzy UP-ideal, fuzzy strongly UP-ideal) of $A$ for all $\beta \in[0, \ddagger]$.

Proof. It follows from Theorems 2.34 and 3.3 (resp. Theorems 2.36 and 3.6, Theorems 2.38 and 3.8, Theorems 2.40 and 3.10).

Theorem 3.28. If there exists $\beta \in[0, \ddagger]$ such that the fuzzy $\beta$-translation $\mu_{\beta}^{\ddagger}$ of $\mu$ is a fuzzy UP-subalgebra (resp. fuzzy UP-filter, fuzzy UP-ideal, fuzzy strongly $U P$-ideal) of $A$, then the fuzzy $\alpha$-translation $\mu_{\alpha}^{\dagger}$ of $\mu$ is a fuzzy UP-subalgebra (resp. fuzzy UP-filter, fuzzy UP-ideal, fuzzy strongly UP-ideal) of A for all $\alpha \in[0, \dagger]$. 
Proof. It follows from Theorems 3.5 and 2.33 (resp. Theorems 3.7 and 2.35, Theorems 3.9 and 2.37, Theorems 3.11 and 2.39).

\section{Conclusions}

In the present paper, we have introduced the concepts of fuzzy $\alpha$-translations of a fuzzy set of type I and of fuzzy $\beta$-translations of a fuzzy set of type II in UP-algebras and investigated some of its essential properties. Finally, we have important relationships between two types of fuzzy translations of a fuzzy set. We think this work would enhance the scope for further study in this field of fuzzy sets. It is our hope that this work would serve as a foundation for the further study in this field of fuzzy sets in UP-algebras.

Acknowledgement. The authors wish to express their sincere thanks to the referees for the valuable suggestions which lead to an improvement of this paper.

\section{REFERENCES}

[1] Akram, M., Bifuzzy ideals of K-algebras, WSEAS Trans. Math., 7 (2008), 313-322.

[2] Akram, M. and Dar, K. H., T-fuzzy ideals in BCI-algebras, Internat. J. Math. E3 Math. Sci., 18 (2005), 1899-1907.

[3] Akram, M. and Dar, K. H., Fuzzy ideals of K-algebras, An. Univ. Craiova Ser. Mat. Inform., 34 (2007), 11-20.

[4] Ansari, M. A. A. and Chandramouleeswaran, M., Fuzzy translations of fuzzy $\beta$-ideals of $\beta$-algebras, Int. J. Pure Appl. Math., 92 (2014), no. 5, 657-667.

[5] Bhowmik, M. and Senapati, T., Fuzzy translations of fuzzy subalgebras in BG-algebras, $J$. Math. Inform., 4 (2015), 1-8.

[6] Chandramouleeswaran, M., Muralikrishna, P. and Srinivasan, S., Fuzzy translation and fuzzy multiplication in BF/BG-algebras, Indian J. Sci. Technol., 6 (2013), no. 9, 5216-5219.

[7] Dar, K. H. and Akram, M., On a K-algebra built on a group, Southeast Asian Bull. Math., 29 (2005), 41-49.

[8] Gerla, G. and Scarpati, L., Extension principles for fuzzy set theory, Inf. Sci., 106 (1998), 49-69.

[9] Hashemin, M. A., Fuzzy translations of fuzzy associative ideals in BCI-algebras, Br. J. Math. Comput. Sci., 16 (2016), no. 1, 1-9.

[10] Hu, Q. P. and Li, X., On BCH-algebras, Math. Semin. Notes, Kobe Univ., 11 (1983), 313-320.

[11] Iampan, A., A new branch of the logical algebra: UP-algebras, Manuscript submitted for publication, April 2016.

[12] Imai, Y. and Iséki, K., On axiom system of propositional calculi, XIV, Proc. Japan Acad., 42 (1966), no. 1, 19-22.

[13] Iséki, K., An algebra related with a propositional calculus, Proc. Japan Acad., 42 (1966), no. 1, 26-29.

[14] Jun, Y. B., q-fuzzy subalgebras of BCK/BCI-algebras, Sci. Math. Jpn. Online, 4 (2001), 197-202.

[15] Jun, Y. B., On $(\alpha, \beta)$-fuzzy ideals of BCK/BCI-algebras, Sci. Math. Jpn. Online, 7 (2004), 101-105. 
[16] Jun, Y. B., Translations of fuzzy ideals in BCK/BCI-algebras, Hacet. J. Math. Stat., 40 (2011), no. 3, 349-358

[17] Jun, Y. B., Roh, E. H. and Kim, H. S., On fuzzy B-algebras, Czechoslovak Math. J., 52 (2002), no. 127, 375-384.

[18] Keawrahun, S. and Leerawat, U., On isomorphisms of SU-algebras, Sci. Magna, 7 (2011), no. 2, 39-44.

[19] Lee, K. J., Jun, Y. B. and Doh, M. I., Fuzzy translations and fuzzy multiplications of BCK/BCI-algebras, Commun. Korean Math. Soc., 24 (2009), no. 3, 353-360.

[20] Lele, C., Wu, C., Weke, P., Mamadou, T. and Njock, G. E., Fuzzy ideals and weak ideals in BCK-algebras, Sci. Math. Jpn. Online, 4 (2001), 599-612.

[21] Prabpayak, C. and Leerawat, U., On ideals and congruences in KU-algebras, Sci. Magna, 5 (2009), no. 1, 54-57.

[22] Senapati, T., Bhowmik, M., Pal, M. and Davvaz, B., Fuzzy translations of fuzzy $H$-ideals in BCK/BCI-algebras, J. Indones. Math. Soc., 21 (2015), no. 1, 45-58.

[23] Sitharselvam, P. M., Priya, T. and Ramachandran, T., Anti Q-fuzzy KU-ideals in KUalgebras and its lower level cuts, Int. J. Eng. Res. Appl., 2 (2012), no. 4, 1286-1289.

[24] Somjanta, J., Thuekaew, N., Kumpeangkeaw, P. and Iampan, A., Fuzzy sets in UP-algebras, Ann. Fuzzy Math. Inform., 12 (2016), no. 6, 739-756.

[25] Zadeh, L. A., Fuzzy sets, Inf. Cont., 8 (1965), 338-353. 